\title{
Experimental research regarding static rigidity in transversal direction of "normal" module
}

\author{
Dragoş-Florin Chitariu* \\ Technical University “Gheorghe Asachi” of Iasi, Prof. Dr. Doc. D. Mangeron Str., 67, Iaşi, Romania
}

\begin{abstract}
The paper presents some experimental results regarding the total deformation of "normal" modules found in the structure of modular sets. The study presents the main aspect regarding the experimental methodology used for the determination of static rigidity in the transversal direction, the measuring scheme and test stand used. Modules deformations were analysed, under the action of transversal static force, in the presence of axial pretension force, using advanced equipment. The research conducted showed that modules with symmetric structure present increased stiffness compared to the asymmetric modules and $\mathrm{T}$ cross channels.
\end{abstract}

\section{Introduction}

In a technological system fixtures are used for orientation-positioning-clamping/tightening of workpieces during machining, measuring, assembly. Modular fixtures are characterized by the use of components that are normalized, standardized. The notion of modularity is related to determining a dimensional correlation based on module and by using multipliers various dimensions can be set. By non-permanent assembly, various fixtures can be built for clamping various workpieces, in various machining, measuring, assembly conditions [1-2]. Modular fixtures have the advantage of high flexibility, reduced assembly times. The main disadvantages: lower technical performance than special permanent fixtures and high cost caused by high geometrical accuracy imposed to modules that are comprise in a modular fixture and the assembled fixture rigidity is lower as a result of a high number of joints [3-6].

The notion of system rigidity (stiffness), in general, therefore of technological systems also, represents the system capacity to oppose deformation under the action of exterior forces [5], [7].

The rigidity (stiffness) of a regular or a modular fixture can be defined as the ratio of the force (or torque) acting on the fixture, in a particular direction, and the deformations (linear or angular) of the structure, in the same direction or not [5].

The rigidity is an important fixture requirement; in order assure the dimensional/ geometrical precisions, during machining, inspection, assembly, especially for difficult machining and severe geometric conditions $[1,2,3,5]$

Static stiffness offers information for evaluating the accuracy of machining process and for evaluation of "resistance" to vibration. Both static and dynamic rigidity/ stiffness, of a

\footnotetext{
*Corresponding author: chitariudragos@gmail.com
} 
modular fixture, is determined by the number, arrangement and static stiffness of the modules within the fixture structure $[4,8]$.

Based on the forces acting on the modular fixture-workpiece system during clamping and machining and decomposition of the structure the following joints may be considered:

- joints axially tensioned (on normal direction);

- joints transversal tensioned, with axial pretension (on normal direction);

- joints torque tensioned, with axial pretension (on normal direction).

Static stiffness analytic determination is difficult due to multiple joints present in the structure and limited information regarding the static behaviour of these joints and, in addition, the surfaces of the modules that compose the modular structure are accompanied by a series of geometrical deviations (dimensional, shape, relative position, waviness, roughness) which can lead to a series of deviations of orientation-positioning and clamping deviations during clamping and machining $[1,3,5,8,9]$.

In the case of joints transversal tensioned, with axial pretension (on normal direction) the information in the Machine-tools and Fixtures fields are limited, compared to the information regarding joints axially tensioned (on normal direction) in the form of values for contact rigidity between the workpiece and supports/ clamping elements without values for the total (overall) rigidity (consisting of contact rigidity and intrinsic rigidity) of fixtures components which compose the structure $[1,2,8,9,10,11]$.

In the paper a work methodology is proposed in order to experimentally determine the values for the total deformation of modules structures, found in modular fixture, which can lead to geometrical deviations caused by static transversal forces in the presence of pretension static forces on axial (vertical) direction of modules and modular structures - assembly forces. Also in this paper, the proposed modules (Figure 3), will be associated with a base plate (Figure 2).

In the case of modular fixtures, frequently, modules that form the fixture body are used for orienting and positioning of workpieces, thus justifying the study of this kind of modules.

\section{Method}

The developed research methodology is based on the knowledge from the Machinery construction field [7, 11], Machine tools field [9], and fixture field [1, 2, 3, 4, 5, 10].

The research methodology contains the following stages:

1. Identification of the influence factors on modular fixture deformation on transversal direction in the presence of assembly forces (on normal direction). Among the factors that may influence the transversal deformations of modular devices we considered: 1-the acting forces (assembly forces, clamping forces, machining forces); 2-module material; 3-clamping fittings; 4-surface roughness of the joint contact surfaces, 5 -number of joints found in the structure; 6-orientation and positioning of the module in the modular structure of the fixture related to the base plate; 7-number of clamping points of the base plate on the machine tool table; 8-module type and size; 9-clamping force [2, 3, 4, 5].

2. Selection of constant and variable factors. The constant factors, which can influence the transversal deformations of modular devices in the presence of axial assembly forces, considered are: axial assembly forces, module type; orientation and positioning of the module in the modular structure of the fixture. The variable factors considered are: transversal force: type of modules, surface on which the transversal force acts.

3. Development of force and deformations measuring scheme and the selection of the force and displacement measuring method. In order to determine the rigidity on transversal direction, in the presence of axial assembly forces, the methodology requires the measurement of the acting force and the fixture displacements. 
Figure 1 presents an illustration of the experimental stand designed developed and used to determine the stiffness of modular fixtures [4].

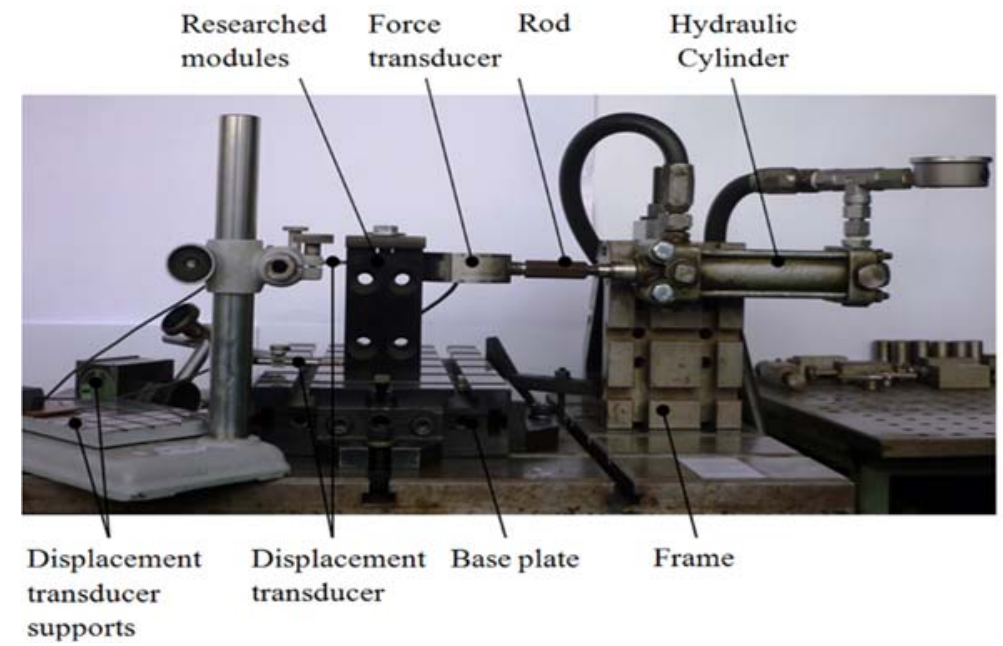

Fig. 1. Test stand designed and developed to determine the stiffness of modular fixtures [4].

The acting force is measured with the force transducer and simulates the effect of the clamping and machining forces. The force transducer is positioned horizontally at $16 \mathrm{~mm}$ to the top surface of the module. The force is applied horizontally by a hydraulic cylinder; deformations were measured at $16 \mathrm{~mm}$ to the top surface and at $16 \mathrm{~mm}$ near the base plate of the modular structure as presented in Figure 1.

The measuring equipment consists of: force transducer CTL model 1000 produced by Laumas Elettronica srl; inductive displacement transducer type $\mathrm{Wl} / 10 \mathrm{~mm}$ produced by Hottinger Baldwin Messtechnik; Spider 8 multi-channel electronic PC measurement unit produced by Hottinger Baldwin Messtechnik; computer [3, 4, 5].

Data processing was performed by filtration using the "moving average" filter. The static force considered was composed from: assembly forces, tightening forces and the static component of the machining forces.

The tested modular structures consisted of modules (elements) from the modular kit SEM - 64 DISROM, made in Romania,. General characteristics of the SEM - 64 DISROM modular kit are: basic dimensions (distance between channels) - $64 \mathrm{~mm}$, channel width -14 mm diameter of threaded holes - M12 and M6, diameter of screws and bolts - M12 and M6, holes $-\varnothing 13 \mathrm{~mm}[3,4,5]$. Histogram 6 presents dimensions and shape of tested modules.

The "normal" module means that the modules section dimensions are $64 \times 76 \mathrm{~mm}$. The "narrow" module means that the modules section dimensions are $64 \times 38 \mathrm{~mm}$, Fig. 2. These modules generally fulfill (most common) the functions of body components and orientationpositioning of workpieces.

The material for the modules is $38 \mathrm{MoCrA109}$, which after heat treatment and nitrating treatment, acquires a surface hardness of $880-1100 \mathrm{HV}$. Active surface roughness $R a$ is between 0.4 and $0.8 \mu \mathrm{m}[3,4,5]$.

Measurements of the displacements and forces were made on the transversal direction at a force of $550 \mathrm{daN}(5.5 \mathrm{kN})$. Data processing and plotting of diagrams was conducted in NI Diadem software. The axial force used for assembly force had the value $36.140 \mathrm{~N}$ (3.641 daN). 


\section{Results}

Diagrams regarding the evolution of deformations (stiffness) are presented in Fig. 2, the results refer to modules associated with the base plate.

Considering the asymmetric construction of modules the acting forces were applied on the $64 \mathrm{~mm}$ and $76 \mathrm{~mm}$ side, resulting different sides that oppose the transversal forces.

In order to correctly orientate and position the modules on to the baseplate guide keys were used during testing.

Figure 2 presents the measured maximum deformation for support "normal" module CSN -003 module (filtered experimental result), at maximum height and minimum height for the second set of determination.

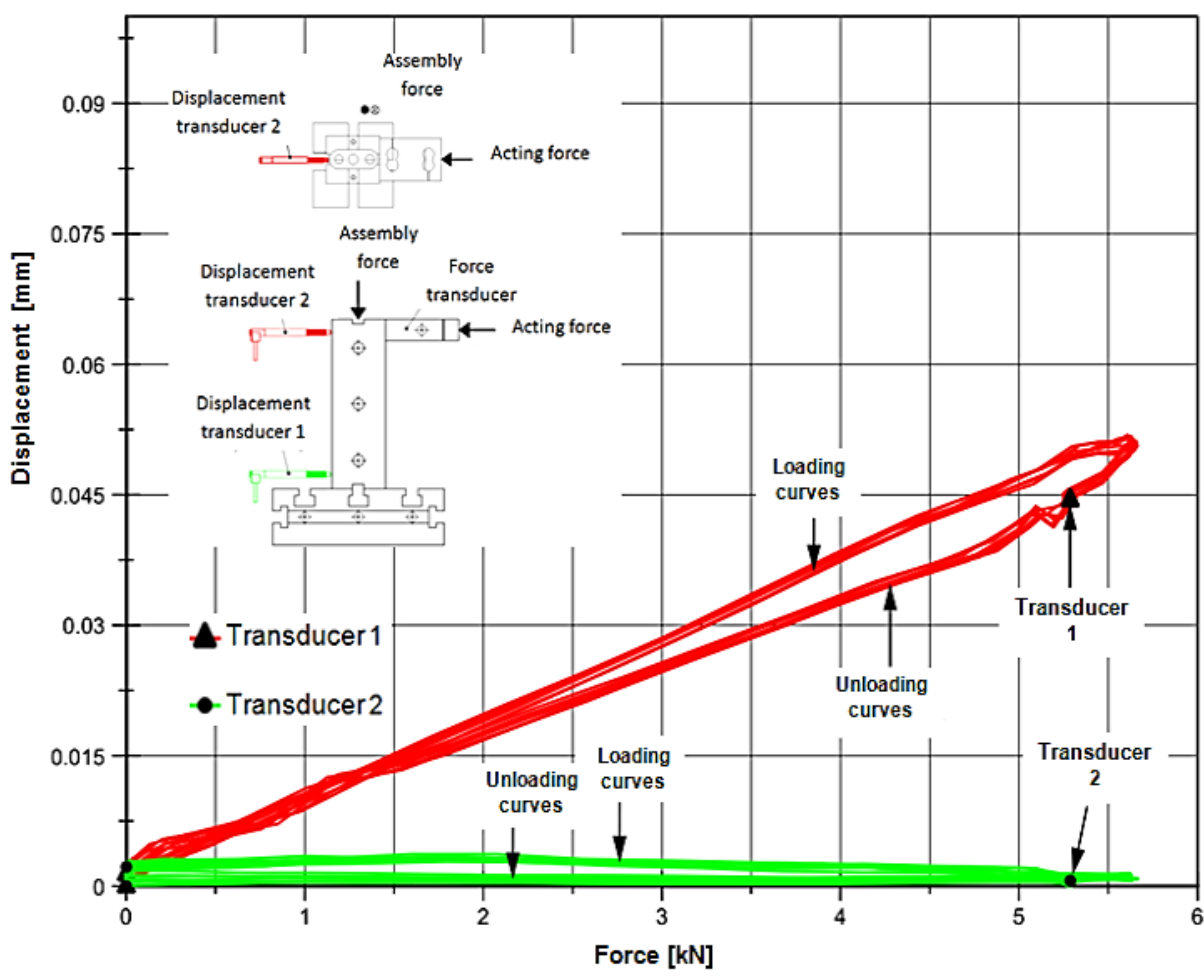

Fig. 2. The deformation (rigidity) curves for support "normal" module CSN -003 module (filtered experimental result).

The maximum measured displacement is $0.0519 \mathrm{~mm}$ for an acting force of $5.56 \mathrm{kN}$.

The maximum measured displacement at the base of the modular structure is $0.0031 \mathrm{~mm}$ for the same force.

The curves shapes are non-linear, aspect specific to joint structures, as presented in literature $[9,10,11]$. The allure of the deformation curves show inflection points, specific to the application of hydraulic actuation force, respectively, the "hydraulic shock" similar to the behaviour presented in $[3,4,6$,$] .$

Figure 3 presents, synthetically, as a histogram, the maximum deformation of modular structures considering acting force of $5.5 \mathrm{kN}$, on transversal direction and a $36140 \mathrm{~N}$ assembly force on axial direction. The acting force was on the $64 \mathrm{~mm}$ and the $76 \mathrm{~mm}$ side. 


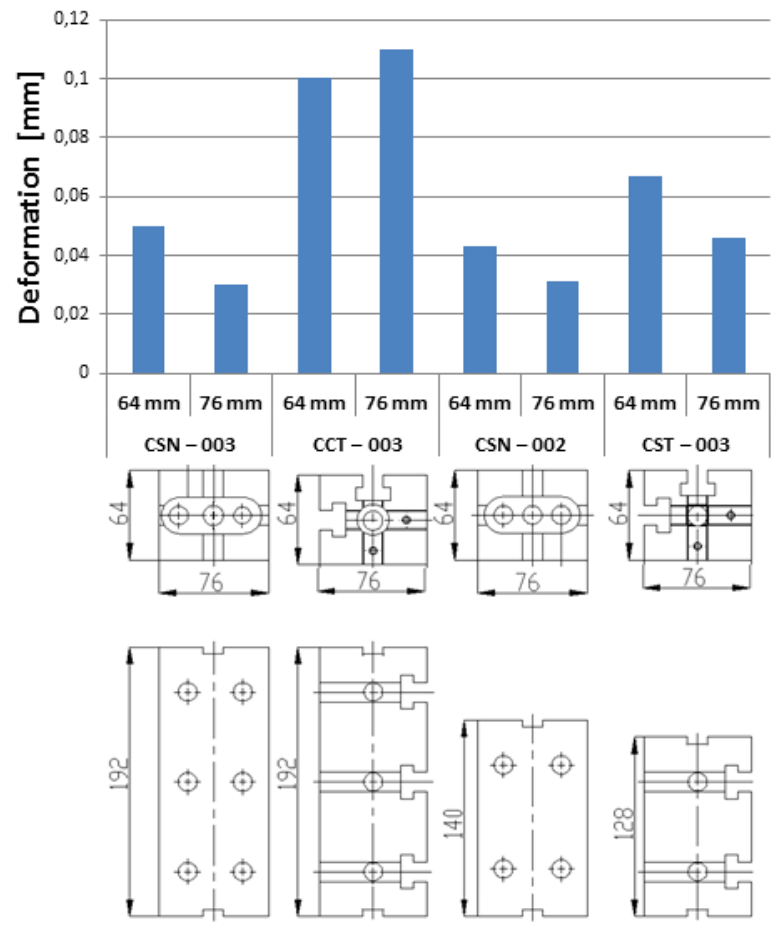

Fig. 3. Maximum deformation considering a $5.5 \mathrm{kN}$ transversal force for the considered structures.

The presented results are after the reduction of the gap caused by the clearance in the wedge assembly. In order to reduce the gap first loading-unloading cycle is performed.

After the first loading cycle and the reduction of gap/ clearance, in order to correctly evaluate the elastic deformations, the measuring equipment was reset to zero a second cycle was performed, without interventions on the system, resulting the graph from Figure 2.

Table 1. Rigidity values calculated for the top of the structure (measured by transducer 2).

\begin{tabular}{|c|c|c|}
\hline \multirow{2}{*}{ Module type } & \multicolumn{2}{|c|}{ Rigidity value at the top of the module [daN/mm] } \\
\cline { 2 - 3 } & $76 \mathrm{~mm}$ side & $64 \mathrm{~mm}$ side \\
\hline CCT -003 & 4.867 & 7.500 \\
\hline CCT -002 & 12.613 & 18.333 \\
\hline CSN -003 & 11.120 & 12.883 \\
\hline CSN -002 & 17.741 & \\
\hline
\end{tabular}

From Figure 3 analysis it is observed that the measured deformations on $64 \mathrm{~mm}$ side (the $76 \mathrm{~mm}$ side is opposing deformation) are reduced compared to deformations when the force acts on $76 \mathrm{~mm}$ side (the $64 \mathrm{~mm}$ side is opposing deformation). Measured deformations are lower by $10-40 \%$ when the acting force is applied on $76 \mathrm{~mm}$ side. 
The rigidity values calculated based on Figure 2 values indicate high rigidity value at the base of the module around 110000 [daN/mm], for all modules.

During testing guide keys were used to orientate and position the modules on the base plate. The absence of guide keys can lead to rotations of the modules under transversal acting force.

\section{Conclusions}

The research conducted has focused on determining the transversal stiffness of modular structure modules constructed from "normal" module from SEM - 64 DISROM modular set. A series of deformation (stiffness) curves were drawn.

Higher stiffness values have been measured for modular structure with modules which do not have cross - channels and which have a symmetric structure. The experimental results indicates a tilting/ leaning of the "normal" module, after the displacements of the entire modular structure alongside the base plate, corresponding to the play between the wedge and the T slot, similar to the results presented for "narrow" [4].

The highlighted displacements and deformations of the modular structure can lead during clamping and machining to "dimensional"/ position deviations from the intended position of the workpiece affecting the machining accuracy.

The research will be extended in the future for more complex structure, typical structures found in this field, in order to asses modular fixture rigidity in common working conditions.

\section{References}

1. N. Gherghel, Fixture Design and Exploitation (in Romanian), 1 (I.P. Iaşi, 1981).

2. N. Gherghel, N. Seghedin, Conception and Design of Supports of Technological Fixtures (in Romanian), (Tehnopress, Iaşi, 2006).

3. D.F. Chitariu, App. Mec. and Mat. 657, 514-518 (2014)

4. D.F. Chitariu, App. Mec. and Mat. 809-810, 754-759 (2015).

5. D. Chitariu, N. Gherghel, Bul. Inst. Polit. Iasi LIX (LXIII), Fasc. 1, $101-110$ (2013).

6. D. Chitariu, N. Gherghel, Bul. Inst. Polit. Iasi LIX (LXIII), Fasc. 1, $111-118$ (2013).

7. V.S. Korsakov, Machining Accuracy (in Romanian, translation from Russian) (I.D.T., Bucharest, Romania 1963).

8. Y. Rong, Y. Zhu, Computer Aided Fixture Design, (Marcel Dekker Inc., New York, 1999).

9. Y. Ito, Modular design for machine tools (The McGraw-Hill Companies, New York, 2008).

10. V. Tache, A. Brăgaru, Fixture for machine-tools, Design of Orientation and Positioning Schemes of Workpieces, (Ed. Tehnica, Bucharest, Romania, 1976).

11. C. Picoş et. al., Design of Machining Technologies, Design Manual, 1 (in Romanian), (Ed. Universitas, Chisinau, Moldova, 1992). 